\title{
Circulación monetaria de la plata en la Hispania del siglo III d.C.
}

\author{
LuIs Sagredo San Eustaquio*
}

Augusto en su reforma monetaria sitúa al denario en 1/84 de libra ${ }^{1}$, es decir con $3,89 \mathrm{~g}$. de peso ${ }^{2}$, proporción que se mantiene hasta la devaluación llevada a cabo por Nerón, en el año 64, que reduce la pieza hasta $1 / 96$ de libra ${ }^{3}$.

El numario, constituido por las piezas de plata, comprendido entre los reinados de Galba y Cómodo, fue muy numeroso, disminuyendo su ley poco a poco durante el siglo II, lo cual será un síntoma del desequilibrio económico que se hará evidente durante el siglo III, como muy bien señala Sutherland ${ }^{4}$.

La ley del denario emitido por M. Aurelio era de 759 por $100^{5}$, que desciende a 725,2 por 100 con Cómodo ${ }^{6}$; encontrándose la primera caída importante durante el primer año de reinado de Septimio Severo alcanzando los 716,7 por $100^{7}$, siguiendo su descenso hasta llegar a los 473,5 por $100^{8}$ durante la segunda etapa de gobierno del primero de los Severos, esto es de 197 a 211.

* Universidad de Valladolid.

$327,45 \mathrm{~g}$.

2 M. Grant: Roman Imperial Money, Edinburgo 1954, pág. 51; C.H.V. SutherLand, Monnaies Romaines, Friburgo 1974, pág. 130.

${ }^{3}$ M. Grant: Roman Imperial..., op. cit., pág. 167; H. MatTingly: Roman Coins from the Earliest Time to the Fall of the Western Empire, Londres, 1928, pág. 123.

${ }^{4}$ C. H. V. Sutherland: Monnaies Romaines..., op. cit., pág. 212.

5 J. GueY: "L'aloi du denier romain de 177 à 211 après d.C.", RN., VI, 4, 1962, pág 117.

6 J. GUEY: “L'aloi du denier romain...", op. cit., pág. 122.

7 J. GuEY: "L'aloi du denier romain...", op. cit., pág. 124.

8 J. GueY: “L'aloi du denier romain...", op. cit., pág. 139. 


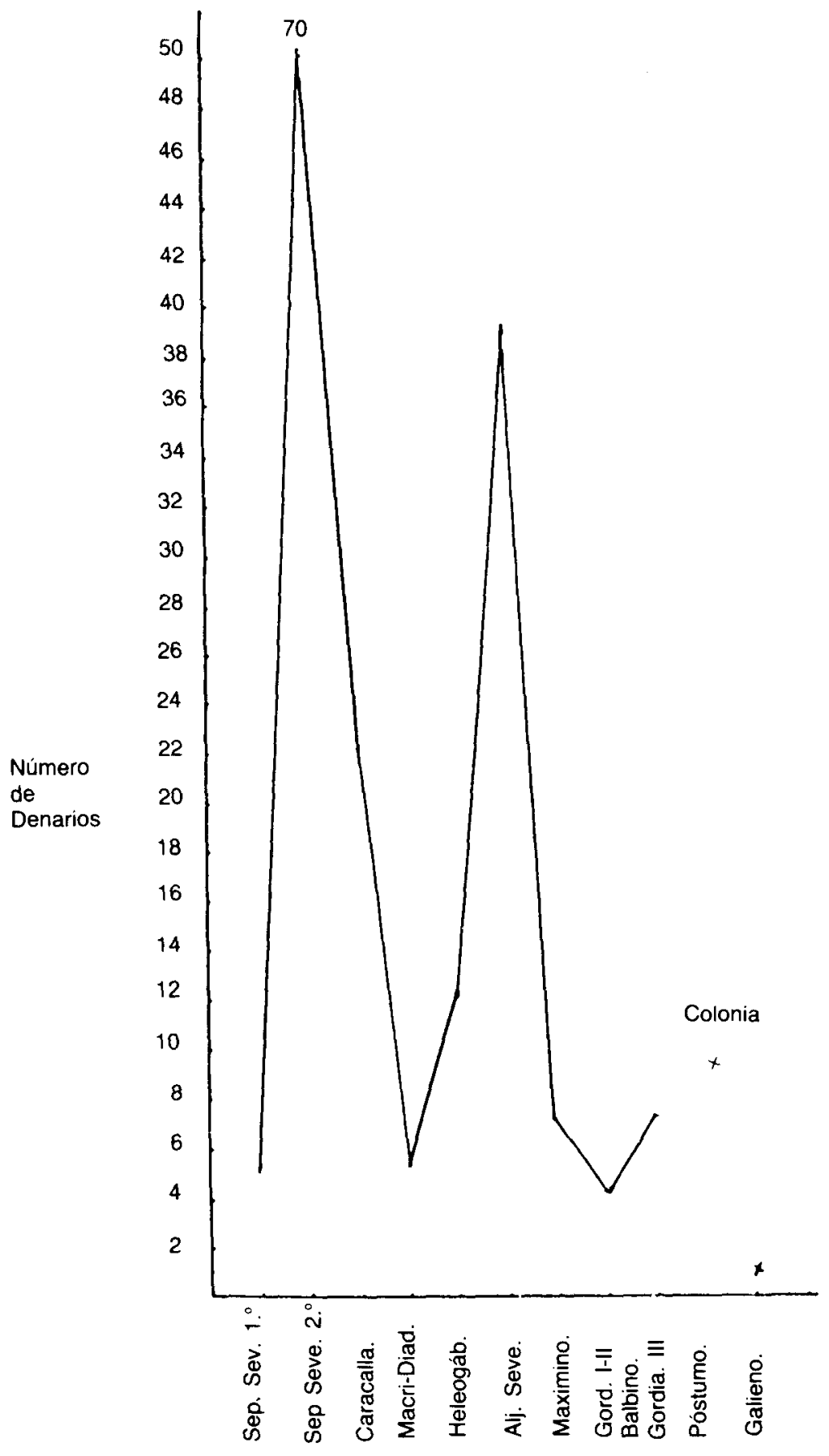


Analizando la evolución del peso del denario desde M. Aurelio observamos que hay un claro descenso durante el reinado de Cómodo, en torno a los $0,30 \mathrm{~g} .{ }^{9}$, en relación con los emitidos durante el período de M. Aurelio. Por el contrario, si lo ponemos en relación con el peso medio correspondiente a la primera etapa del reinado de Septimio Severo (193197), es el período de guerras, es de recuperación ${ }^{10}$, estando en torno a los 0,20-0,30 g., es decir, que vuelven durante el segundo período de gobierno ${ }^{11}$ aproximadamente al peso que tenían durante la época de $\mathrm{M}$. Aurelio.

Este tipo de denario constituido durante el período de Septimio Severo permanecerá estable durante 20 años. Su peso, según Guey, comenzará a disminuir a partir de Heliogábalo ${ }^{12}$, lo que no ocurre en nuestro estudio, como veremos más adelante.

El origen de la devaluación monetaria llevada a cabo en esta época, pensamos que fue como consecuencia de la disminución de las existencias de metales preciosos, debida a una balanza comercial deficitaria que explicaría en parte las pérdidas sufridas por las monedas tanto en su ley como en su peso ${ }^{13}$, asi como por la incidencia que tuvieron las guerras con sus enormes gastos ${ }^{14}$ y la necesidad de numerario como consecuencia de la urbanización y transformación de la economía rural ${ }^{15}$.

Para el estudio de la distribución y evolución del denario en la Península lbérica realizaremos tres cortes cronológicos: el primero, comprenderá todo el período de los Severos (193-235); el segundo, las emisiones realizadas entre los años 235 y 253 , o etapa final del denario y el tercero, corresponderá a la segunda mitad del siglo III, que bien pudiéramos llamar etapa testimonial del denario.

El número total de denarios con que contamos para la realización de nuestro estudio es de 183.

9 J. GuEY: “L'aloi du denier romain...", op. cit., págs. 117 y 122

10 J. GUEY: "L'aloi du denier romain...", op. cit., pág. 124.

"J. GUEY: "L'aloi du denier romain...", op. cit., pág. 129.

12 J. GueY: "L'aloi du denier romain...", op. cit., pág. 92.

${ }^{13} \mathrm{M}$. CORTI: «Note di numismatica economica. Decadenza della moneta nel III secolo dell'Impero Romano", Numismatica, 18, 1942, págs. 24-27; A. H. M. JonEs: "Inflation under Roman Empire", EcHR., II 53, 1953, págs. 293-294; R. THOMSEN: Ille Conferénce International d'Histoire Economique, Munich, 1965, III, págs. 109-110.

${ }^{14}$ M. Grant: Roman imperial..., op. cit., pág. 247 y 251; J. P. Callu: La Pólitique Monétaire des Empereurs Romains de 238 à 311, Paris, 1969, págs. 322-323.

${ }_{15}$ P. OLiva: "Zum Problem der Finanzkrise im 2. und 3 Jahrhundert u.z. römischen Reich", Des Altertum, 8, 1962, 1, págs. 39-46. 


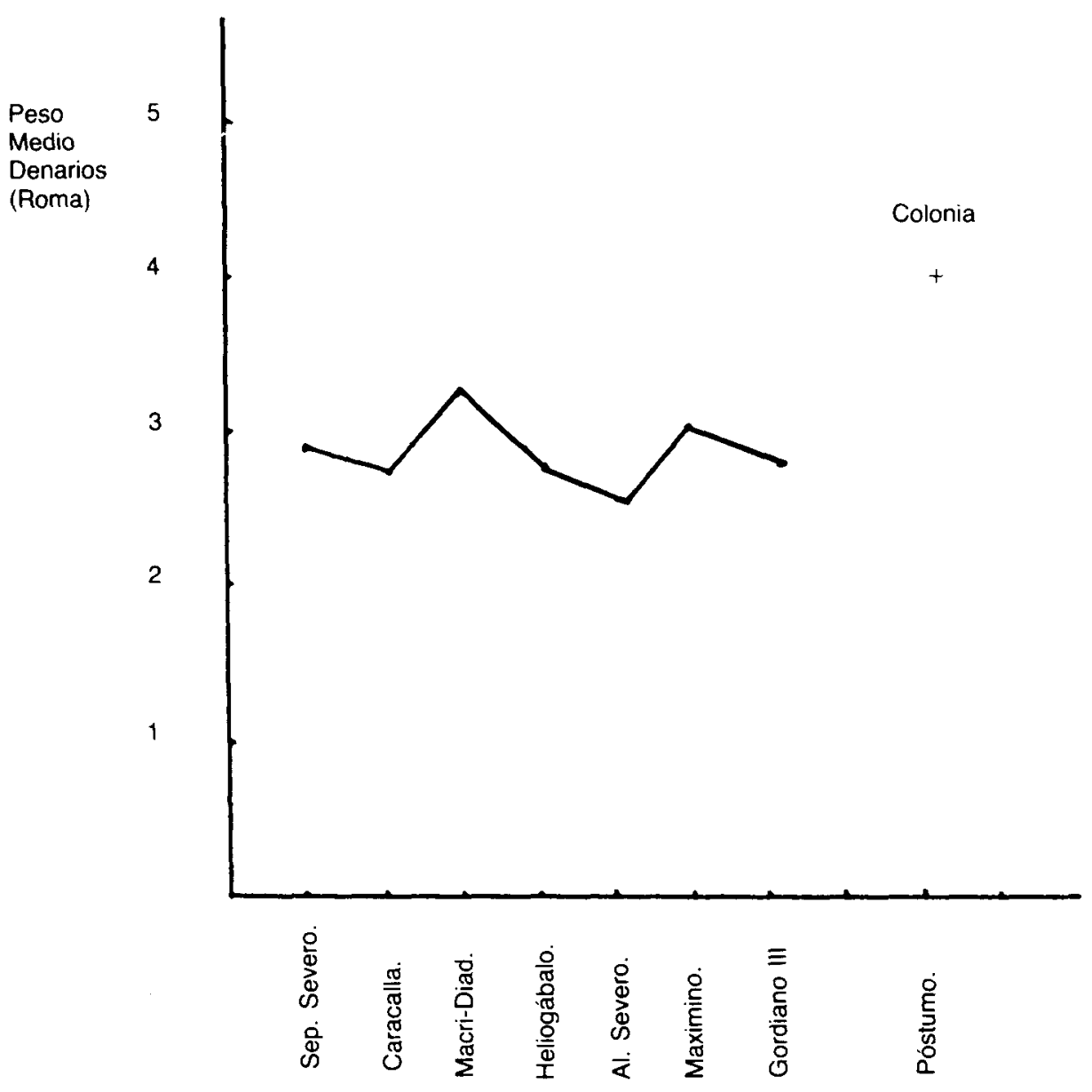

De los 183 denarios, 155 ejemplares corresponden al período de gobierno de la dinastía Severa y equivalen a un 84,69 por 100 . Es decir, que representan casi la totalidad de este tipo de piezas, que fueron emitidas a lo largo de 42 años, lo que supone una emisión de 3,69 muestras por año.

\section{ÉPOCA SEVERA (193-235)}

De las 155 monedas pertenecientes a esta etapa dos de ellas son ilegibles, por lo que a la hora de realizar las proporciones y los porcen- 
tajes por emperadores prescindiremos de ellas, así pues trabajaremos con 153 ejemplares.

Los denarios emitidos durante el reinado de Septimio Severo son 75 , equivalentes al 48,38 por 100 de los emitidos durante esta etapa.

El estudio de las piezas de este emperador to realizaremos en dos apartados: el primero, corresponde a los años de las luchas por el poder, es decir, 193-197, y el segundo, de 197 a 211.

Al primer período pertenecen cinco monedas, lo que supone un 6,66 por 100 de las piezas de este emperador, de ellas tres fueron emitidas por la ceca de Emesa y las otras dos por el taller de Roma.

El peso medio de los ejemplares acuñados por la ceca oriental es de $2,88 \mathrm{~g}$., lo que está por debajo de la norma ${ }^{16}$. Estas muestras fueron emitidas en los últimos momentos de las luchas contra Pescenio Níger ${ }^{17}$.

El segundo período comienza al concluir las guerras internas con la victoria de Septimio Severo en 197, llegando hasta el año 211 momento en que muere el emperador.

A esta etapa corresponden 70 monedas, lo que equivale a un 93,24 por 100 de las emitidas durante todo el reinado. Estos ejemplares fueron emitidos 48 en Roma, el 96 por 100 y dos en Laodicea, con un 4 por 100.

El peso de las piezas acuñadas en Roma es de 2,91 g., lo que está por debajo de la media ${ }^{18}$ como en el caso anterior; esto nos indica que estas monedas tuvieron un alto indice de circulación que redundó en el desgaste del material constituyente de la composición.

Los ejemplares emitidos en Laodicea nos dan un peso medio de 3,08 g., mayor y más equilibrado que los anteriores encontrándose por debajo de las dos tablas de confrontación ${ }^{19}$, que es de $3,18 \mathrm{~g}$., la una y de $3,11^{20}$, la otra.

El porcentaje de las piezas emitidas por año de reinado es de 4,16 ejemplares. $3 \mathrm{~g}$.

${ }^{16}$ J. GueY: «L'aloi du denier romain...", op. cit., pág. 127, 3,109 g., HCC., III, pág. XVII,

17 P. PETIT: L'Empire Romain, Paris, 1974, pág. 327.

${ }^{18}$ BMC., V, 3,18 g.; J. GuEY: “L'aloi du denier romain...", op. cit., pág. 138, 3,11 g.

${ }^{19}$ HCC., III, pág. XVII, 3,02 g.

${ }^{20} B M C$, , V, pág. $\mathrm{XX}, 3,16 \mathrm{~g}$. 


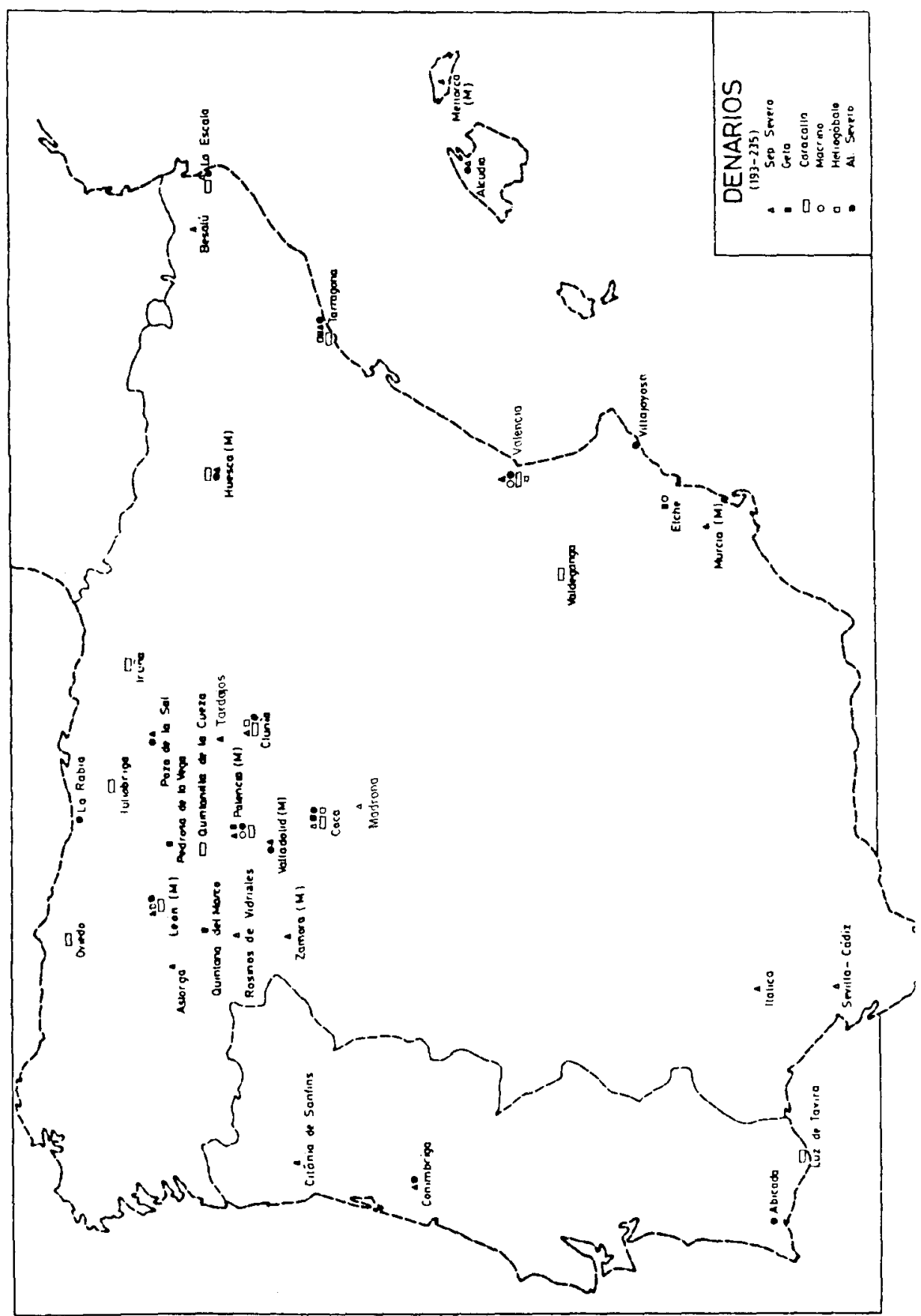


Circulación monetaria de la plata en la Hispania del siglo II d.C.

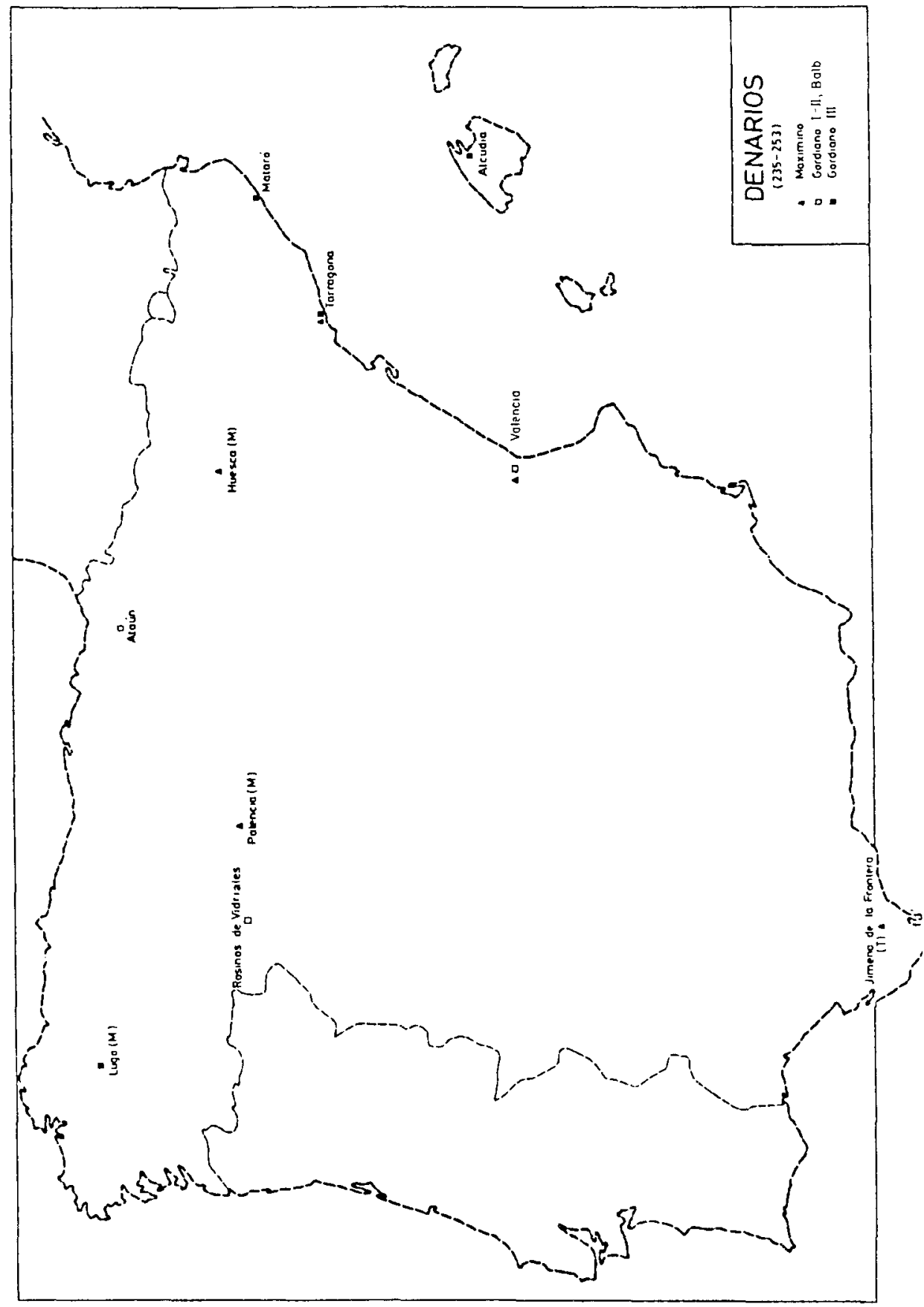


La distribución de las monedas en la geografía peninsular nos da dos grandes zonas de acumulación como son: la Meseta Norte, con un 36 por 100 y Cataluña, con un 34,66 por 100, siguiendo a continuación la región levantina, con un 13,33 y otras situadas a gran distancia de las primeras, por su escasa concentración de material, como son las Baleares y Portugal, con un 4 por 100, encontrándose seguidamente Aragón y Andalucia, con un 2,66 por 100, terminando con Cantabria y Navarra, con un sólo ejemplar cada una, lo que representa un 1,33 por 100 .

Caracalla ${ }^{21}$ está representado por 22 ejemplares, lo que representa un 14,37 por 100 de los emitidos durante la etapa que estamos estudiando. Todos ellos fueron acuñados en el taller de Roma.

El peso medio de estas piezas es de $2,68 \mathrm{~g}$. que está por debajo de las equivalentes en las tablas de comparación que utilizamos ${ }^{22}$. El número de numismas emitidos por año es de 3,66.

El reparto de estas piezas es similar al de la etapa anterior, es decir, en primer lugar el grupo más numeroso se encuentra acumulado en Cataluña con un 36,36 por 100 , seguido de la Meseta Norte y Levante con un 22,72 por 100 y por último, todas con una pieza y un 4,54 por 100 Aragón, Navarra, Asturias y Cantabria.

De Macrino y Diadumeniano tenemos cinco monedas, tres del primero y dos del segundo, todas ellas acuñadas en Roma, siendo su peso medio de $3,05 \mathrm{~g}$. lo que está por debajo de la media ${ }^{23}$.

La emisión de monedas por año es de 5 numismas, centrándose su distribución en la Península en dos puntos exclusivamente, en Levante y en la Meseta Norte, con un 80 por 100 y un 20 por 100 respectivamente.

Heliogábalo ha dejado constancia de su presencia mediante las 12 muestras que nos han llegado, todas ellas emitidas en el taller de Roma, con excepción de una que lo fue en una ceca oriental, Antioquia.

El peso medio de los ejemplares procedentes de Roma es de 2,67 grs., lo que nos indica que no llega a la media ${ }^{24}$, mientras que el peso de la muestra oriental es de 2,75 g., igualmente por debajo de la

21 Únicamente utilizamos las monedas emitidas durante su reinado personal, ya que las acuñadas como César y Augusto con Septimio Severo son incluidas en la etapa de reinado de éste.

22 BMC., V, pág. XX, 3,10 g.; HCC., III, pág. XVIII, 3,06 g.

${ }_{23} B M C$., V, pág. XXI, 3,10 g.; HCC., III, pág. XVIII, 3,11 g.

${ }^{24} B M C$., V, pág. XXI, 2,98 g.; HCC., III, pág. XVIII, $3 \mathrm{~g}$. 
norma ${ }^{25}$. La cantidad de monedas emitidas por año es de tres, siendo la dispersión en el espacio geográfico relativamente pequeña ya que se encuentra concentrada en tres zonas, en la Meseta Norte, con el 41,66 por 100 , en Cataluña, con el 33,33 por 100 y en la región levantina, con el 25 por 100 .

El último emperador de este período es Alejandro Severo que con 39 numismas es el que mayor cantidad de ejemplares nos ha dejado tras Septimio. Todos ellos fueron emitidos en la ceca de Roma, teniendo como peso medio 2,40 g., lo cual se encuentra por debajo de la media que seguimos ${ }^{26}$.

La emisión de monedas por año es de tres; siendo su distribución de las muestras en el territorio hispano de mayor amplitud que en casos precedentes siendo la región de mayor acumulación la Meseta Norte, con un 48,71 por 100; seguida a continuación de la zona catalana, con un 23,07 por 100; seguidamente tenemos a Portugal con un 12,82 por 100; luego Levante, con un 10,25 por 100 y por último Aragón y Baleares con un 2,56 por 100 respectivamente.

\section{PERIODO DE LOS AÑOS 235-253}

A esta etapa corresponden 18 denarios, lo que supone un 9,83 por 100 , lo cual nos indica claramente la extraordinaria recesión que sufre la acuñación de este tipo de moneda. La proporción de monedas/año que tiene lugar a lo largo de este período es de 1,38, la cual no podemos tener en cuenta ya que después de Gordiano III se deja de emitir regularmente en casi su totalidad ${ }^{27}$.

Al emperador Maximino pertenecen siete ejemplares, lo que supone un 38,88 por 100 de las muestras de este período, todas emitidas por un taller de Roma. Su peso medio es de $2,77 \mathrm{~g}$. peso que se encuentra como en casos anteriores por debajo de la media que seguimos ${ }^{28}$. El

${ }^{25} B M C$., V. pág. XXI, 2,94 g.; HCC., III, p. XXIII, 2,99 g.

${ }^{26} B M C .$, VI, pág. 2,99 g.; HCC., III, p. XIX, 3,02 g.

${ }_{27} \mathrm{~J}$. P. CALLU: "Les monnaies de compte et le monnayage du bronze entre 253 et 295", CIN., (Roma 1961), 1965, pág. 372; HCC., III, pág. XII.

${ }^{28} B M C$., VI, 3,01 g.; HCC., III, pag. XIX, 3,07 g. 


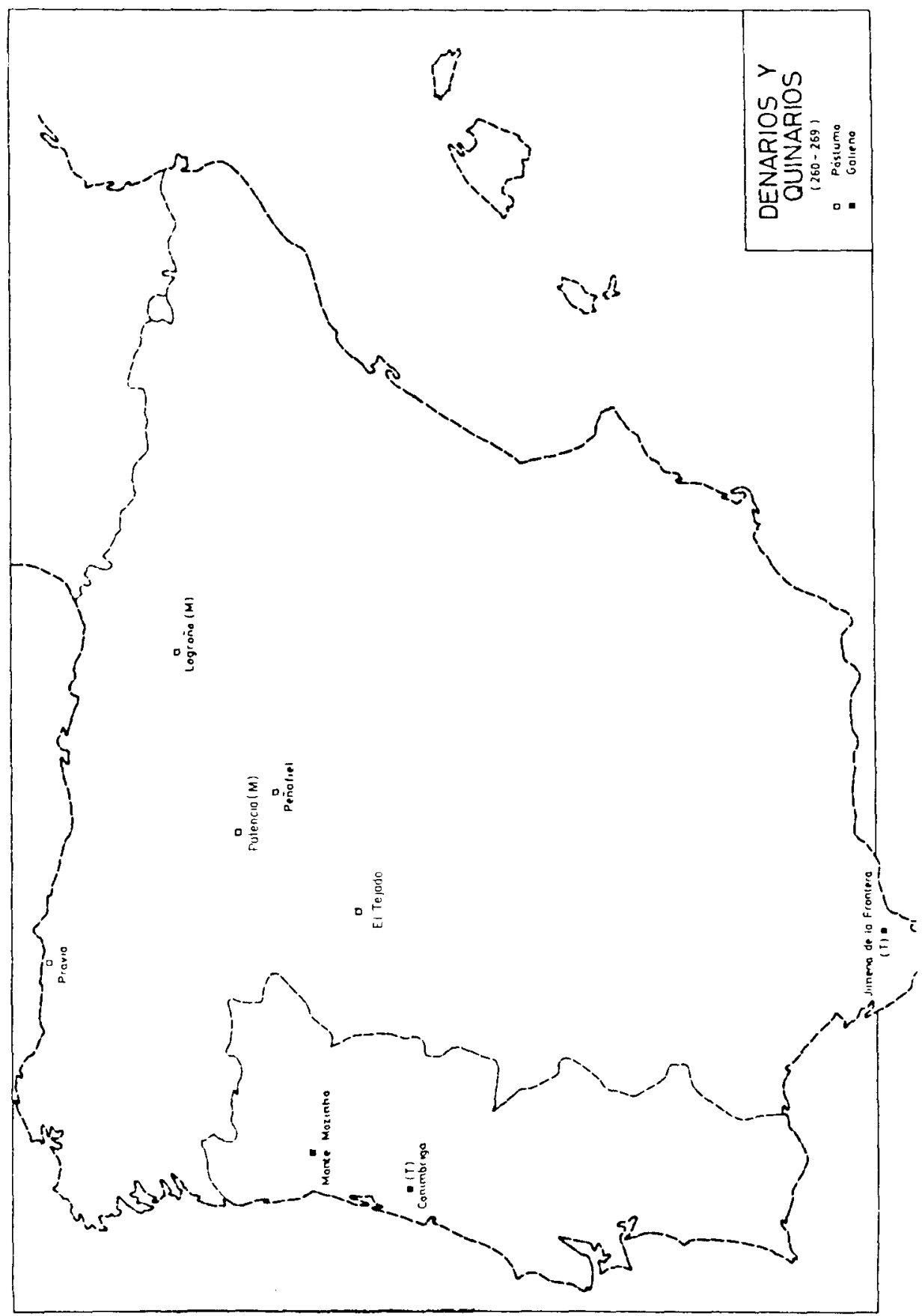


porcentaje de monedas por año, durante el reinado de este emperador, es de 2,33 piezas.

Los distintos numismas se distribuyen por cuatro zonas; correspondiendo a Cataluña el mayor porcentaje, un 57,14 por 100, seguida por las otras tres, Aragón, Levante y Meseta Norte, con un 14,28 por 100 respectivamente.

Las monedas siguientes cronológicamente a las de Maximino son las pertenecientes a Gordiano I, Gordiano II y Balbino, a las cuales tomamos en conjunto al constituir una misma fase, que está representada por cuatro piezas de plata, de éstas hay una, la de Gordiano II, de la que no tenemos ni peso ni leyenda, y en su hallazgo tuvo lugar en la provincia de Guipúzcoa, en Ataún. El peso medio de las restantes es de 2,66 g.

Gordiano III, es el emperador considerado como el último acuñador de denarios ${ }^{29}$, puesto que con Filipo I son muy raras las muestras de este tipo ${ }^{30}$. Tenemos siete ejemplares correspondientes a este soberano, lo cual supone un 38,38 por 100 de las piezas pertenecientes a esta etapa, siendo todas ellas acuñadas en la ceca de Roma. El peso medio de estas monedas es de 2,92 g., media que se encuentra por debajo, pero muy próxima a la media normal ${ }^{31}$. En función del material con que contamos tenemos una proporción monedas/año de 1,16. La distribución de estos numismas en la Península se centra fundamentalmente en Cataluña, donde hay un 57,14 por 100, a la que sigue Baleares, con un 28,57 por 100 , siendo el otro lugar donde se localizan en el Noroeste, con un 14,28 por 100 .

\section{PERIODO DE LOS AÑOS 253-285}

Durante esta época este tipo de monedas es muy escaso, tanto los denarios como los quinarios fueron acuñados principalmente en ocasiones especiales como son los aniversarios imperiales, matrimonios, ascen-

${ }^{29}$ RIC., IV-3, p. XXII: C.H.V. SUTHERLAND: “Denarius and Sestertius in Diocletian's Coinage Reform". JRS., 1961, pág. 94.

${ }_{30}$ J. P. Callu: "Les monnaies de compte...", op. cit., pág. 372; C. H. V. Sutherland: Monnaies Romaines...,op. cit., pág. 25.

${ }_{31}$ RIC., IV-3, p. XXII, 2,93 g.; HCC., III, pág. XIX, 2,98 g. 
sión de un César, el nacimiento de un heredero y, sobre todo, en los aniversarios celebrados cada cinco años. Estas piezas eran acuñadas y distribuidas como obsequio del emperador a los miembros de la corte, del ejército e incluso entre el pueblo ${ }^{32}$. Las piezas de esta etapa son muy raras y escasas, no tenemos más que aquellas que fueron emitidas por el Imperio Galo y Galieno.

El único y dudoso quinario que tenemos pertenece a la época del reinado personal de Galieno, fue acuñado en el taller de Roma y tiene un peso de $0,86 \mathrm{~g}$., que está por debajo de la norma ${ }^{33}$; fue hallado en Portugal, en Monte Monzinho. No obstante, teniendo en cuenta su módulo, nos inclinamos a pensar que tal vez sea un denario, aunque en muy mal estado ${ }^{34}$. Pensamos por ello que pudiera ser un error considerarlo un quinario ${ }^{35}$.

Los últimos denarios con que contamos pertenecen al Imperio Galo, habiendo sido emitidos por Póstumo.

Son ejemplares de mala calidad, aunque tienen un peso de 3,76 gramos, peso que está ligeramente por debajo de la media ${ }^{36}$. Las nueve monedas fueron emitidas por la ceca de Colonia y fueron halladas en la franja norte de la Península, en la provincia de Palencia, el 55,55 por 100 ; en la de Valladolid, el 22,22 por 100 y el 11,11 por 100 respectivamente en la Rioja y en la de Salamanca.

\section{DENARIOS HALLADOS EN TESORILLOS}

Un elemento que hay que tener en cuenta es el de la aparición de denarios en los distintos tesorillos encontrados en la Península, y de esta

${ }^{32}$ P. Strauss: "Les monnaies divisionnaires de Trèves après la réforme de Dioclétien", RN., V, 16, 1, 1954, págs. 21-22; R. MAC MULLEN: "The Emperors Largesses", Latomus, XXI 1, 1962, págs. 159-166; C. E. KING: "Denarii and Quinarii A.C. 253-295", Scripta Numaria Romana, Essays Presented to Hunphrey Sutherland. Londres, 1978, págs. 79-80.

${ }^{33}$ HCC., IV, XIV; C. E. KING: “Denarii and Quinarii...", op. cit., pág. 89.

${ }^{34}$ Módulo $16 / 18$ y peso $0,86 \mathrm{~g}$.

${ }^{35}$ R. M. S. CENTENO: "As moedas", en Escavaçoes no Monte Monzinho, II, 1975-1976 Penafiel, 1977, pág. 45, n. 28.

${ }^{36}$ HCC., IV, pág. XIV, $3,78 \mathrm{~g}$. 
manera ver la convergencia o divergencia de la evolución del denario en ambos casos.

El total de estas piezas aparecidas en los tesorillos es de 20 ejemplares; 14 de estos, el 70 por 100 , pertenecen a la época Severa, correspondiendo 13 de ellos, un 92,85 por 100, al reinado de Septimo Severo, de los cuales dos, el 15,38 por 100, pertenecen a la primera etapa de su gobierno, habiendo sido acuñado uno de ellos en el taller de Emesa y el otro en el de Roma.

Los pesos medios de estos ejemplares son de 2,44 g. para el de Emesa, enconírándose por debajo de los pesos medios normales ${ }^{37}$ mientras que el peso de la pieza coétanea emitida en Roma, es de 2,34 g., encontrándose igualmente por debajo de la media ${ }^{39}$.

Las 11 monedas emitidas durante la segunda etapa del reinado de Septimio Severo representan el 84,61 por 100 del total de su período de gobierno; siendo el peso medio de estas muestras de 3,44 g., lo cual nos indica que se encuentran por encima de los tipos medios ${ }^{39}$, así como del de los hallazgos sueltos, que hemos visto más arriba ${ }^{40}$. Todas estas piezas fueron emitidas en la ceca de Roma.

Otro ejemplar acuñado igualmente durante la época Severa, y que representa el 7,69 por 100 , pertenece a la época del gobierno personal de Caracalla; su peso es realmente importante, puesto que con sus 3,79 g. está, como los anteriores, por encima tanto de los pesos medios de las tablas de comparación ${ }^{41}$, como del correspondiente de los hallazgos sueltos ${ }^{42}$.

Todas estas muestras emitidas durante la época Severa han sido halladas en tres tesorillos ${ }^{43}$, que aparecieron, dos, en la meseta Norte, en Coca y Rosinos de Vidriales, $y$, en un tercero, en Monforte de la provincia de Alicante.

${ }^{37}$ BMC., V, pág. XX, 3,12 g.; HCC., III, pág. XVIII, $2,88 \mathrm{~g}$.

${ }^{38} B M C .$, V. pág. XX, 3,10 g.; HCC., III, pág. XVII, 3,14 g.

${ }^{39}$ BMC., V, pág. XX, 3,15 g.; HCC., III, pág. XVII, $3,14 \mathrm{~g}$

${ }_{40}$ El peso medio de los hallazgos sueltos es de $2,91 \mathrm{~g}$.

4' BMC., V. pág. XX, 3,10 g.; HCC., III, pág. XVIII, 3,13 g.

${ }^{42}$ El peso medio de los hallazgos sueltos es de $2,68 \mathrm{~g}$.

43 L. SAGRedo: "Posible tesorillo del siglo Il d.C." Numisma, XXXI, 168-173, 1981, págs. 73-88; R. MARTín VALLS, G. DeliBes: «Hallazgos arqueológicos de la provincia de Zamora" (IX), BSAA., XLVIII, 1982, pág. 362. R. ARROYO: “Tesorillo de monedas tardorromanas de Monforte (Alacant), ActaNum., 15, 1985. 
Al segundo periodo en que hemos dividido el estudio y que corresponde a los años 235-523, pertenecen únicamente dos muestas, equivalentes al 10 por 100 del total, emitida una a nombre de Paulina ${ }^{44}$ y la otra acuñada por Gordiano $1{ }^{45}$; teniendo esta última un peso de 3,07 gramos, valor que se encuentra muy próximo a la norma que seguimos ${ }^{46}$. La primera de estas monedas fue hallada en el sur de Hispania ${ }^{47}$ y la segunda en la Meseta Norte ${ }^{48}$.

De la tercera y postrera etapa de nuestro estudio, que tiene lugar entre los años 253-285, tenemos cuatro ejemplares que suponen el 20 por 100. Dos de ellos fueron hallados formando parte del mismo tesorillo ${ }^{49}$, en el de Jimena de la Frontera y otro en la zona de Portugal ${ }^{50}$; estas tres piezas fueron emitidas durante la etapa del gobierno personal de Galieno. El último numisma pertenece al Imperio Galo y fue acuñado por Póstumo ${ }^{51}$, siendo su lugar de hallazgo en el norte de Hispania, en Asturias ${ }^{52}$.

Los denarios correspondientes a Galieno son los únicos hallados en Hispania ${ }^{53}$ y aparecen sumergidos dentro de una gran cantidad de antoninianos, resultando ser un mero símbolo de constatación de existencia. El peso conocido es de 1,21 g., el cual se encuentra por debajo de la media ${ }^{54}$.

La pieza de plata acuñada por Póstumo fue hallada en el norte de la Península ${ }^{55}$, tiene un peso de $3,75 \mathrm{~g}$., y se encuentra por debajo de la media de los hallazgos sueltos ${ }^{56}$, lo mismo que ocurre en su comparación con las tablas que utilizamos ${ }^{57}$.

${ }^{44}$ H. D. GallweY: "A hoard of Third Century Antoniniani from Southern Spain", NC., 1962, pág. 362.

${ }_{45}$ R. Martin Valls; G. Delibes: «Hallazgos arqueológicos...”, op. cit., pág. 61.

${ }^{46} B M C$., VI, págs. 247-248, 3,19 g.; HCC., III, pág. XIX, 3,09 g.

47 Jimena de la Frontera (Cádiz).

${ }^{48}$ Rosinos de Vidriales (Zamora).

49 H. D. GallweY: “A Hoard of Third Century...", op. cit.., págs. 390 y 403.

so I. Pereira; P. Bost; J. Hiernard: Fouilles de Conimbriga III. Les Monnaies. París 1974 , pág. 25.

51 M. Mallo VIESCA: "Tesorillo de denarios romanos de D. ${ }^{a}$ Palla", Archivum, XVI, 1966, págs. $273,3,75$.

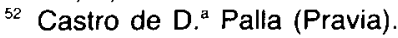

${ }^{53}$ Cfr. nota 34.

${ }^{54}$ C. E. KING: “Denarii and Quinarii..”, op. cit., pág. 89.

${ }_{55}$ Castro de D. ${ }^{a}$ Palla (Pravia, Asturias).

${ }^{56}$ El peso medio de las piezas sueltas es de $3,97 \mathrm{~g}$.

57 HCC., IV, pág. XIV, 3,78 g. 


\section{CONCLUSIONES}

La evolución del denario durante el siglo III es de un franco descenso tanto en lo referente al metal fino como al peso.

Según Heichelheim ${ }^{58}$ la inflación comienza en época de Cómodo, con la devaluación del denario en torno al 170 por 100 , lo que hace que la moneda, al contrario de lo que había ocurrido durante el siglo II, bien aceptada fuera de las fronteras del Imperio, ahora sea tomada con desconfianza. No obstante, no hay pruebas que consoliden la existencia real de esta crisis durante el reinado de Cómodo ${ }^{59}$, observándose además que las acuñaciones de este emperador se encuentran en tesorillos posteriores, lo que nos da a entender que sí eran aceptados ${ }^{60}$.

Todo esto nos hace ver que la crisis comienza con Septimio Severo, ya que es en realidad cuando se observa claramente, entre otras cosas, un aumento importante del número de tropas, y por tanto de la moneda de plata ${ }^{61}$, ya que está relacionada directamente con el pago de las soldadas al ejército. Esta caída tuvo lugar a comienzos del año $194^{62}$, apenas un año después de su elevación al trono ${ }^{63}$ redujo drásticamente el fino del metal, llegando el contenido de plata a un porcentaje inferior al 50 por $100^{64}$. Esta reducción de la plata del denario comportaba una serie de ventajas financieras para el emperador, ya que con dos denarios "buenos", podian acuñarse tres de los "nuevos" ${ }^{65}$, piezas que posteriormente empezaron a ser rechazadas ${ }^{66}$.

58 F. HeIChelheim: "Zur Währungskrisis des römischen Imperiums im 3 Jahrhundert", Klio, 26, 1933, págs. 102 y 113.

59 T. PEKARY: «Studien zur römischen Währungs und Finanzgeschichte von 161 bis 235 n. Chr.", Historia, 8, 1959, págs. 446 y 470.

60 S. BOLIN: “Fynden av romerska mynt i det fria Germanen», BRGK., 19.

61 A. PASSERINI: "Gli aumenti del soldo militare de Comodo a Massimino", Athenaeurn, 1946, págs. 145-159.

62 J. GUEY: «Peut-on se fier aux essais chimiques?: encore l'aloi romain de 177 à 211 après J.C.". RN., VI, 7, 1986, pág. 177.

63 R. O. FINK; A. HOEY; W. SNYDER: “Feriale Duranum», YCIS., VII, 1940, pág. 257.

64 J. GuEY: “L'aloi du denier romain...", op. cit., págs. 83,87 y 90; J. GuEY: “Peut-on se fier aux essais chimiques?...", op. cit., págs. 120-121.

65 M. MAzzA: Lotte sociali e restaurazione autoritaria nel III Secolo d.C., Laterza, 1973, pág. 294.

${ }^{66}$ S. Bolin: State and Currency in the Roman Empire to 300 A.D., Estocolmo 1958, págs. 212 y ss., y 248 y ss. 
Esta reducción del metal fino, y paralelamente el aumento, de la masa monetaria, era el procedimiento de devaluación de la moneda de plata que desembocó en la gran inflación, que tuvo lugar durante los últimos años del reinado de Galieno y a lo largo del de Claudio II.

El momento clave de la depreciación del denario tuvo lugar, como hemos dicho más arriba, en 194; a partir del 195 y hasta el 311 tenemos una etapa de estabilidad, para posteriormente comenzar a disminuir el peso imparablemente ${ }^{67}$. Más adelante, en la Galia, Póstumo acuñó una serie de denarios mucho más pesados que los de Galieno ${ }^{68}$, y también más numerosos.

A través de los materiales analizados de los hallazgos sueltos, hemos podido comprobar que la mayor cantidad de piezas emitidas tiene lugar durante el período Severo con el 84,69 por 100. En este momento vemos que el mayor número de ejemplares pertenece a Septimio Severo, con el 41,43 por 100 seguidamente tenemos un fuerte descenso durante el gobierno de Caracalla que llega hasta el 12,15 por 100, continuándose la caída con Macrino-Diadumeniano, con un 2,76 por 100 . Hay una ligera recuperación con Heliogábalo alcanzando un 6,62 por 100 y con Alejandro Severo se llega incluso a una cota superior, 27,35 por 100, cota que no vuelve a conseguir como consecuencia de la fuerte devaluación que tuvo lugar durante este reinado ${ }^{69}$, que dio lugar a que el pueblo aceptara cada vez menos esta moneda, inclinándose por la de bronce ${ }^{70}$.

Este descenso se continúa con Maximino llegando hasta un 3,82 por 100 , para bajar aún más durante el espacio cronológico en el que gobiernan los Gordiano I y II, así como Balbino y Pupieno, en el que se consigue un 0,54 por 100; seguidamente con Gordiano III hay un mínimo impulso ascendente que se remonta hasta el 3,82 por 100, esto es el canto del cisne, ya que a partir de este momento los denarios desaparecen, manteniéndose algunas acuñaciones y en pequeñas cantidades, este es el caso de la muestra que tenemos de Galieno.

Pertenecientes al Imperio Galo poseemos nueve ejemplares de Póstumo, los cuales nos indican la escasa implantación que debió de tener en la Península, centrándose ésta además en el Norte de la Península.

67 J. P. Callu: La politique monétaire..., op.cit., pág. 238

68 P. BAStien: "Travaux d'Hercule dans la monnayage de Postume", RN., VI, 1, 1958, págs. 59-78.

69 J. GuEY: "La devaluation du denier romain sous Septime Sévère", BSAF., $1952-$ 1953, págs. 89-91 y en BSFN. 1960, págs. 400, 413, 422, 455 y 457.

70 R. TuRCAN: Le trésor de Guelma. Etude Historique et Monétaire, Paris 1963, pág. 47. 
En el peso medio de los denarios vemos que desde Septimio Severo hay un descenso continuado hasta llegar a Alejandro Severo, iniciándose una ligera recuperación con Maximino y posteriormente con Gordiano III que marca la cota más alta de los pesos medios desde Alejandro Severo, con excepción, claro está, de los pesos medios de las piezas emitidas por Póstumo que se encuentran por encima de la media.

El hecho de que los denarios emitidos en Roma tengan un peso menor a la media, es debido a que tuvieron un mayor tiempo de circulación y también a que de salida tuvieron un peso inferior a los emitidos en Oriente.

Las piezas son acunadas todas en Roma, con excepción a los tres ejemplares de la primera etapa de Septimio Severo, que fueron emitidos por el taller de Emesa, y dos de la segunda que lo fueron en Laodicea. Hasta la época de Póstumo todas las muestras que poseemos se trabajaron en la ceca de la metrópoli, siéndolo las de este último emperador en el taller de Colonia. Las monedas mencionadas en primer lugar, las orientales, pudieron llegar a Hispania a través de los soldados del ejército al ser trasladados o bien al haber causado baja al alcanzar la edad reglamentaria y como veteranos quedar libres para viajar o bien por relaciones comerciales a pequeña escala y a través de terceros, ya que las mencionadas piezas fueron emitidas por los talleres orientales para el pago de las soldadas a las tropas que luchaban contra Pescenio Níger.

La distribución geográfica de los distintos numismas nos dice que tenemos dos núcleos importantes de acumulación que destacan sobre los demás, la Meseta Norte, con un 36,46 por 100 y Cataluña, con un 30,38 por 100. Levante, Portugal, Baleares y Aragón con un 16,57 por $100 ; 4,97$ por $100,3,31$ por 100 y 2,76 por 100 respectivamente, encontrándose muy por detrás de los puntos principales, estando más alejadas aún Andalucia, Cantabria y Navarra con 1,10 por 100 y Asturias, Guipúzcoa, Noroeste y Rioja con un 0,55 por 100 cada una de ellas. Basándonos en éstos observamos que hay un ligero predominio de material en la zona mediterránea siendo éste el 51,36 por 100 de la masa monetaria. La importancia acumulativa de los puntos principales se debe a que en Cataluña tenemos un importante centro administrativo y en la Meseta Norte, que corresponde aproximadamente con el convento Cluniense, hay un importante centro agrícola-ganadero. Sin embargo, observamos que en la zona sur apenas existe material conocido para nuestro estudio, como consecuencia, tal vez, de la falta de trabajos publicados y de las necesarias excavaciones. 
Como en otras zonas del Imperio, como puede ser Brigetio ${ }^{71}$ los últimos hallazgos de denarios de nuestro trabajo pertenecen a Gordiano III, teniendo sólamente detrás una muestra de Galieno y las correspondientes al Imperio Galo.

Hemos comprobado que las monedas correspondientes a los hallazgos sueltos siguen una evolución similar a la de los denarios aparecidos en los tesorillos, donde hay también un total predominio de los ejemplares acuñados por Septimio Severo, e igualmente tenemos una representación de la ceca oriental de Emesa.

Los últimos numismas pertenecen a Póstumo y Galieno, con un predominio de las piezas de éste sobre el anterior, inversamente a lo que ocurre entre los hallazgos sueltos. Vemos en el conjunto de estas piezas que las de Galieno se encuentran en el sur de Hispania, mientras que las de Póstumo se hallan en la zona norte. En el número total de monedas de estos dos emperadores encontramos una mayor proporción en favor de Póstumo, pero tenemos que recordar que el emperador galo acuñó un número mucho mayor de este tipo de ejemplares; lo que nos indica, en función de estos datos, es que la incidencia gala sobre nuestra Península es mayor que la de Roma, lo cual resulta totalmente erróneo ya que a nivel general de la circulación monetaria, en estos momentos, hay un total predominio de la moneda romana sobre la gala en una proporción francamente arrasadora, un 96,77 por 100 a favor de Galieno por un 3,22 por 100 a favor de Póstumo ${ }^{72}$.

Si en la primera parte de nuestro estudio, los lugares de concentración nos dan como principal punto de acumulación la Meseta Norte, en el caso de los tesorillos el foco más sobresaliente tiene igualmente lugar en esta región, siendo además la zona en que aparecieron todos los ejemplares pertenecientes a la etapa de los Severos, con excepción del de Monforte ${ }^{73}$, apareciendo el de Póstumo en Asturias y los de Galieno en el sur de Hispania y zona de Portugal.

Todo esto nos demuestra que hubo una penetración de la moneda, en este caso el denario, con dirección Este-Oeste, es decir, siguiendo la ruta Cataluña, Aragón y Meseta Norte, con débiles ramificaciones en

$71 \mathrm{~K}$. BIRO-SEY, Coins from Identified Sites of Brigetio and the Question of Local Currency, Budapest, 1977, pág. 56.

${ }^{72}$ L. Sagredo, Circulación e inflación monetaria en la Hispania romana del siglo il d.C., Tesis Doctoral inédita teida en la Universidad de Valladolid, curso 1982-1983, págs. 182 a 285 y págs. 418.425 , págs. 1.606 y 1.615 .

73 R. Arroyo, “Tesorillo de monedas...", op. cit. 
torno a esta vía. Por consiguiente la circulación de moneda de plata, denario, durante el siglo III d.C. es muy semejante a la que tiene lugar con el bronce en la Península ${ }^{74}$, aunque esta última tiene una mayor incidencia en la zona de la Bética.

Otra vía de penetración sería a través de la vía Augusta la cual llevaria el monetario hacia el sur.

Diferenciándose ambas en cuanto a la distribución que tendrá lugar posteriormente con el antoniniano, el cual desplazará el centro de la circulación hacia la mitad norte de Hispania.

A través de todo lo expuesto podemos decir que la Península Ibérica estaba inmersa en un único circuito monetario dependiente de Roma, en lo que al denario se refiere.

${ }^{74}$ L. SAGREDO, «La circulación y desaparición del bronce en la Hispania romana (193285)", Estudios en Homenaje a Don Claudio Sánchez Albornoz, vol. I, Buenos Aires, 1983. págs. 173-222. 


\section{BIBLIOGRAFÍA}

Affonso dos Santos, M. L.: Arqueología Romana do Algarve. Vol. I-II. Lisboa, 1971-1972.

AlfARO, C.: «Monedas con indicación de procedencia recientemente integradas en la sección de Numismática del M.A.N., I", Boletín del MAN, vol. III, 2, 1985, págs. 137-149.

Arroyo, R.: Numerario de la Universidad de Valencia, 1984.

ARroyo, R.: "Tesorillo de monedas tardorromanas de Monforte (Alacant)". ActaNum., 15, 1985, págs. 139-156. 1985.

BaralBar, F.: «Antigüedades de Iruña. Discurso leído en el Ateneo de Vitoria al abrirse el Curso 1882-1883", Euskal-Eriia, IX (1883), 1978.

Belda Dominguez, J.: "Museo Arqueológico Provincial de Alicante. Ingresos procedentes de Torre-La Cruz (Villajoyosa, Alicante)", MMAP, VIII (1947), 1948.

Belda Navarro, C.: El proceso de romanización de la provincia de Murcia. Murcia, 1975.

BLANCO, F. J.: Moneda y circulación monetaria en Coca. Segovia, 1988.

CAMPO, M.: "Contribución al estudio de la circulación monetaria en Menorca durante el Imperio Romano", ActaNum., VI, 1976, págs. 6171.

CAmpo, M.: "Circulación monetaria en la villa de La Olmeda, Pedrosa de la Vega (Palencia)", Symposium Numismático de Barcelona, I, págs. 125-138. Barcelona, 1979.

COLLANTES, E.: «Una muestra de la circulación monetaria en la provincia de Osca", Symposium Numismático de Barcelona, I, págs. 117-124. Barcelona, 1979.

Corominas, J. M. ${ }^{\text {; }}$ Marqués, J.: Catálogo Monumental de la Provincia de Gerona. I, La Comarca de Besalú, Gerona, 1976. 
Chaves, F.: "Avance sobre la circulación monetaria en Itálica", Symposium Numismático de Barcelona, II, págs. 77-86. Barcelona, 1979.

Diego Santos, F.: Historia de Asturias. Asturias romana y visigoda. Oviedo, 1975.

FERNÁNDEZ AlLER, M. ${ }^{a}$ C.: Epigrafía y Numismática romana en el Museo Arqueológico de León. León, 1978.

Fernández Chicarro, C.: «Colección de monedas de plata de época antigua procedente de la Baja Andalucía», MMAP, 1947.

GALLWEY, H. D.: «A Hoard of Third-Century Antoniniani from Southern Spain», NC, 1962, págs. 335-406.

Gómez Moreno, M.: Catálogo Monumental de España. Provincia de León. Madrid, 1925.

GurT, J. M. : Clunia III. EAE., 145. Madrid, 1985.

HIERNARD, J.: "Monedas del siglo IIl en el Museo Arqueológico Provincial de Tarragona», ActaNum. VIII, 1978, págs. 97-133.

MAllo Viesca, M.: «Tesorillo de denarios romanos de Doña Palla». Archivum, XVI, 1966, pág. 273.

MAÑANES, T.: Epigrafía y Numismática de Astorga romana y su entorno. Salamanca, 1982.

Martín Bueno, M.: "Numismática antigua en e! Museo de Logroño", ActaNum. IV, 1974, págs. 65-85.

Martín Valls, R. y Delibes, G.: “Hallazgos arqueológicos en la provincia de Zamora IX", BSAA., XLVIII, 1982, págs. 45-70.

Martinez Santa-Olalla, J.: «Antigüedades romanas de Poza de la Sal (Burgos), Anuario de Prehistoria madrileña, II-III, 1931-1932, págs. 160-167.

MAteU Y LlopIS, F.: “Hallazgos Monetarios VI», Ampurias, XIII, págs. 203-254.

-: "Hallazgos Monetarios IX», NumHisp., II,4, 1953, págs. 275-302.

-: «Hallazgos Monetarios XVII», NumHisp., VIII,15-16, 1959, págs. 151-164.

MiChelenA, L.: "Guipúzcoa en la época romana», BRSVAP, XII, 1956, págs., 75-76.

MUSEO DE LUGO. Estudiado directamente.

MUSEO DE MURCIA. Estudiado directamente.

MUSEO DE PALENCIA. Estudiado directamente.

MUSEO DE.SANTANDER. Estudiado directamente.

Nieto Gallo, G.: El oppidum de Iruña. Vitoria, 1958.

PAÇO, A. DO: “Citania de Sanfins. V, nota sobre algunas Moedas", Nummus, III, 7, 1954.

Pereira, I.: "Achados monetarios de Monte Mozinho", Conimbriga, XIII, 1974, págs. 75-166. 
Pereira, I.; Bost, J. P. y Hiernard, J. P.: Fouilles de Conimbriga. III, Les Monnaies. Paris, 1974.

RIPOLL, E.; NUIX, J. M.; y VILLARONGA, L.: «La circulación monetaria en Emporion», Symposium Monetario de Barcelona, I, págs. 445-455. Barcelona, 1979.

SAGRedo, L.: “La presencia romana en la provincia de Palencia durante el siglo III d.C.", HAnt., IX-X, 1979-1980, págs. 31-56.

-: “Posible tesorillo del siglo III d.C.», Numisma, XXXI, 1981, 168-173, págs. 73-88.

-: Circulación e inflación monetaria en la Hispania romana del siglo III d.C., Tesis inédita leída en la Universidad de Valladolid durante el curso 1982-1983.

-: "La representación imperial romana de los Severos a Diocleciano, en el Museo A.P.", PITTM., 51, 1984, págs. 5-32.

-: "La circulación del áureo en la Hispania del siglo III d.C.", Conimbriga, XXVI, 1986, págs. 89-98.

-: La circulación monetaria romana a través del numario del Museo Arqueológico de Valladolid (en prensa).

SAGReDO, L. y ZUMEL, L.: “Hallazgos numismáticos en la provincia de Valladolid", HAnt., VII, 1977, págs. 301-306.

SAGREDO, L. y ARRIBAS, E.: Circulación y evolución monetaria en la provincia de Segovia en la antigüedad. Segovia, 1987.

SÁNCHEZ JIMÉNEZ, J.: "Inventario de los hallazgos monetarios en la provincia de Albacete", Publicaciones del Seminario de Historia y Arqueología, Albacete, 1962, pág. 110.

Silva, A. C. F. DA y Centeno, R. M. S.: "Excavaçoes arqueologicas na Citania de Sanfins (Paços de Ferreira), 1977-1978», Portugalia, I, 1980.

TARDAJOS (Deobrigula). Material inédito en estudio.

Trapote, M. ${ }^{\text {a }}$ C. y Martín Valls, R.: "Hallazgos monetarios en Clunia de 1958 a 1964", BSAA., XXX, 1964, págs. 129-169.

VEGA DE LA TORRE, J. R.: “Numismática antigua de la provincia de Santander", Sautuola, III, 1982, págs. 235-270.

Velasco, V.: Catálogo-inventario del Museo Provincial de Bellas Artes de Zamora. Zamora, 1958.

Viana, A.; Fermosinho, J. y Veiga Ferreira, O. DE: “De lo prerromano a lo árabe», AEA., XXVII, 87, 1953, págs. 113-138. 